\title{
FOS Oncoprotein
}

National Cancer Institute

\section{Source}

National Cancer Institute. FOS Oncoprotein. NCI Thesaurus. Code C17439.

c-FOS protein is a transcription factor critical in regulating the development of the skeleton. C-FOS protein is a major component of the DNA-binding AP-1 transcription complex, which also includes members of the JUN family. Each protein contains a bZIP domain and a leucine zipper domain. FOS oncoproteins derive from mutated FOS genes. These proteins have been found in the Finkel-Biskis-jinkins (FBJ-MSV) and Finkel-BiskisReilly (FBR-MSV) murine sarcoma viruses, which induce osteogenic sarcomas in mice. The FBJ-MSV v-fos gene encodes a 55 kD protein and the FBR-MSV v-fos gene encodes a 75 kD fusion protein. (from OMIM 164810 and $\mathrm{NCl}$ ) 